\title{
Política de Salud Intercultural: Perspectiva de usuarios mapuches y equipos de salud en la IX región, Chile
}

\author{
Ana María Alarcón $\mathrm{M}^{1 \mathrm{a}}$, Paula Astudillo $\mathrm{D}^{\mathbf{2 b}}$, \\ Sara Barrios $\mathbf{C}^{3 \mathrm{c}}$, Edith Rivas $\mathbf{R}^{3 \mathrm{~d}}$. \\ Intercultural health care policy from \\ the perspective of health care \\ providers and mapuche clients
}

Background: Intercultural health is becoming an emergent topic in the design of health care programs for Mapuche people of Chile. This process faces important challenges such as the scarce theoretical support about the meaning of intercultural health and their practical consequences for providers and clients. Aim: To explore the perception in providers and Mapuche clients about intercultural health. Material and methods: A survey performed in 11 counties with the highest concentration of Mapuche people, of the IX region of Chile. The perception about the development of a new health policy specially designed for Mapuche patients was surveyed in 399 Mapuche patients and 64 providers of primary health care centers. Results: Mapuche clients considered, as the main regional challenges, the indifference and discrimination of health care teams towards Mapuche patients, aggravated by the indifference of authorities. Providers considered that the main problem was a lack of knowledge about Mapuche culture and skills to deal with this ethnic group. Patients and providers agreed on the need to use Mapuche dialect in health care attentions, to coordinate actions with traditional healers and to accept ethnical therapeutic practices. Conclusions: There is scarce agreement between providers and Mapuche clients about the need for an special intercultural health policy, its contents, and the regional conditions for its implementation and development (Rev Méd Chile 2004; 132: 1109-14). (Key Words: Ethnic groups; Health care delivery; Indians, South American; Population groups; Quality of health care)
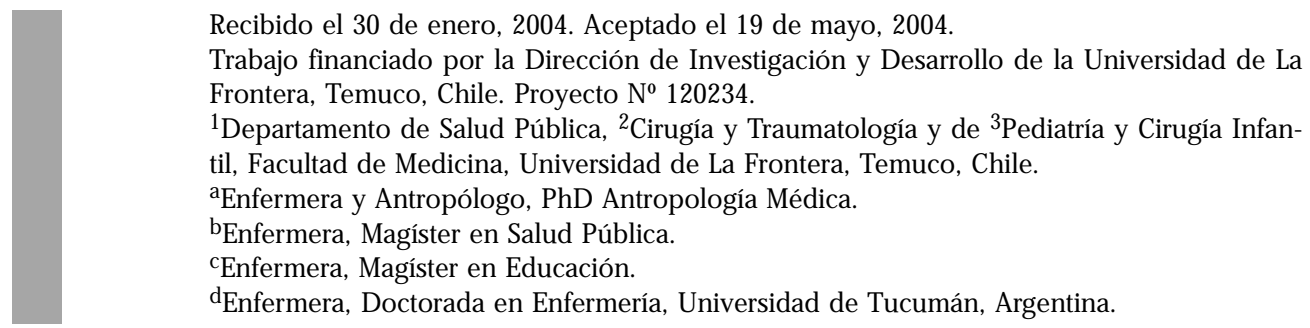

Correspondencia a: Ana M Alarcón M. Departamento de Salud Pública, Facultad de Medicina, Universidad de La Frontera. Montt 112, Casilla 54-D, Temuco, Chile. Fono: (5645) 325738. Fax: (56-45) 325777. E mail: amalarc@ufro.cl 
$\mathrm{E}^{1}$ concepto de Salud Intercultural, ha sido frecuentemente utilizado para identificar la estrategia que considera las variables étnicoculturales de la población indígena en el proceso de atención de salud ${ }^{1,2}$.

La Organización Panamericana de la Salud, en el año 1996, firmó un acuerdo con el Parlamento Indígena de las Américas, en donde estipulaba la preparación de una agenda legislativa para las políticas de salud y la producción de leyes que protegieran a las tradiciones indígenas ${ }^{3}$. En Chile, la interculturalidad en salud se ha traducido en una propuesta de política de Salud y Pueblos Indígenas ${ }^{4}$. Al respecto, se observa que dentro de sus principios fundamentales apunta a la equidad en salud para los pueblos indígenas, quienes viven en situación de pobreza económica y marginalidad social, y a la interculturalidad como proceso de cambio cultural, la cual contribuińa al reconocimiento de la diversidad de modelos médicos de la población indígena ${ }^{4}$. En este contexto, se plantea la necesidad de avanzar hacia la construcción colectiva de un modelo de salud con enfoque intercultural, y a una mayor participación de los pueblos indígenas en los programas de salud.

Lo que dificulta generar un modelo que otorgue una solución culturalmente pertinente a los problemas de salud de la población mapuche, son por un lado, la ausencia de referentes teóricos respecto al significado de la interculturalidad en salud, las implicancias prácticas que tiene el desarrollo de este enfoque para proveedores de salud y usuarios mapuches; y por otro, el escaso conocimiento de las condiciones estratégicas de la región para implementar una política de salud intercultural.

La presente investigación pretende explorar la opinión de proveedores y usuarios mapuches hacia una política de salud que otorgue atención culturalmente apropiada a la población mapuche de la IX región. El análisis se desarrolló en base a los dominios de necesidad y justificación de la política, sus componentes conceptuales y prácticos, principios de gestión para la implementación de una política de salud intercultural y los desafíos regionales para su desarrollo e implementación.

\section{MATERIAL Y MÉTODO}

Estudio descriptivo transversal y comparativo, realizado durante el período 2001-2002 con una muestra probabilística de 463 individuos divididos en: usuarios mapuches $(n=399)$ y proveedores de
Atención Primaria en Salud $(n=64)$ pertenecientes a 11 comunas de mayor concentración mapuche de la IX región: Chol-Chol, Nueva Imperial, Teodoro Smith, Temuco, Padre Las Casas, Freire, Lautaro, Puerto Saavedra, Carahue, Curarrehue, Melipeuco y Lonquimay. En la estimación del tamaño muestral se consideró la proporción de usuarios mapuches inscritos en cada centro de atención primaria seleccionado para el estudio, y de proveedores contratados en dichos centros. Los cálculos estimaron un nivel de confianza de 95\% y un error estándar de 8\%. La condición mapuche de los usuarios fue calificada según registro de dos apellidos mapuches y residencia en comunidades rurales.

En relación a la caracterización de la muestra de usuarios mapuches, el $72 \%$ eran femeninos, $55 \%$ casados, y sus edades fluctuaban entre $18 \mathrm{y}$ 88 años, con un promedio de 41 años. El 93\% se declaró indigente o FONASA A, dueñas de casa y campesinos como trabajo principal. Respecto a la escolaridad, el 59\% tenía enseñanza básica incompleta, el 25\% enseñanza media, y el 16\% estudios técnico-superiores. Respecto al uso de la lengua mapuche el 45,5\% hablaba castellano, 5\% mapuche, y $54 \%$ ambas lenguas.

Respecto a la muestra de proveedores, 50\% fueron profesionales de Atención Primaria con una edad entre 21 y 60 años, y un promedio de 34. La mayor parte estuvo representada por el sexo femenino $(67,3 \%)$.

Para la recolección de información se creó un cuestionario estructurado con preguntas en escalas de 1 (totalmente en desacuerdo) a 5 (totalmente de acuerdo), y dicotómicas el cual contiene cinco dominios representativos del tema salud intercultural. Los cuales surgieron a partir de los resultados de una etapa cualitativa preliminar y corresponden a: 1) Necesidad y justificación de una política de salud intercultural, 2) Concepto de salud intercultural, 3) Elementos simbólicos y prácticos necesarios para el desarrollo de una política de salud intercultural, 4) Principios de gestión para la implementación de la política de salud intercultural, y 5) Desafíos regionales para una política de salud intercultural. El nivel de confiabilidad de los dominios arrojó un Cronbach desde 0,7 a 0,8 .

Estadística. El análisis de los datos comprendió la caracterización y comparación de opiniones de proveedores y usuarios para cada uno de los 
dominios. Se compararon frecuencias y proporciones de las respuestas de ambos grupos mediante ttest $\mathrm{y}$ Wilcoxon. Las diferencias fueron consideradas como significativas para un valor de $p$ $<0,05$. Posteriormente, se realizó un análisis multivariado de componentes principales para cada uno de los dominios. Se utilizó el STATA 8.0 y SPSS 11.

\section{RESULTADOS}

Se presentan en relación a los cinco dominios:

1. Necesidad y justificación de una política de salud intercultural en la IX región. La Tabla 1 muestra la diferencia de opinión entre usuarios mapuches y proveedores. Los primeros están totalmente de acuerdo con la necesidad de una política de salud intercultural que otorgue atención especial a la población indígena, sin embargo, los proveedores no están de acuerdo con esta necesidad (p: 0,00). La misma situación ocurre con la opinión sobre las dificultades de acceso de la población mapuche al sistema de atención de salud. Los usuarios están totalmente de acuerdo en que existen importantes problemas de acceso, lo cual justifica la creación de una política de salud especial para los pacientes mapuches, los proveedores en cambio, no perciben que estos problemas sean mayores al de la población no mapuche de la región ( $\mathrm{p}$ : 0,00 ). El análisis de componentes principales amojó que las dificultades de acceso de la población mapuche explicańa el 77\% de varianza total de las respuestas ( $\alpha$ de Cronbach $=0,70$ ).

2. Concepto de salud intercultural. El concepto de salud intercultural (Tabla 2), en el que concuerdan tanto proveedores como usuarios, se expresa en la incorporación de facilitadores interculturales y de la lengua mapuche en el sistema de atención de salud, en la integración de prácticas terapéuticas de la medicina mapuche, y en la incorporación de agentes tradicionales al sistema de atención dirigido a los usuarios mapuches. Opiniones divergentes se manifiestan en relación a la necesidad de otorgar atención de salud especial a la población mapuche; la mayonía de los usuarios está de acuerdo con esta concepción, no así los proveedores (p: 0,00). El análisis de componentes principales mostró que el concepto de salud intercultural se explica a través de dos componentes. El primero con $78 \%$ total de varianza $(\alpha=0,76)$ señala que integrar las prácticas de la medicina mapuche e incorporar activamente a los agentes tradicionales de salud son indicadores del concepto de salud intercultural. El segundo componente con

Tabla 1. C ondiciones de la IX región para una Política de Salud Intercultural. D istribución de la opinión de acuerdo a proveedores y usuarios en la IX región

\begin{tabular}{|lcccccccc|}
\hline \multirow{2}{*}{ Condiciones } & \multicolumn{3}{c}{ Usuario } & \multicolumn{3}{c}{ Proveedor } & \multirow{2}{*}{ P } \\
& Moda & $\bar{x}$ & DS & Moda & $\bar{x}$ & DS & \\
\hline Necesidad de una política & 5 & 4,1 & 1,1 & & 2 & 2,6 & 1,3 & 0,00 \\
Acceso del usuario al sistema de atención & 5 & 3,9 & 1,2 & & 2 & 2,9 & 1,4 & 0,00 \\
& & & & & & & & \\
\end{tabular}

Tabla 2. Concepto de salud intercultural. D istribución de la opinión de acuerdo a proveedores y usuarios en la IX región

\begin{tabular}{|lrrrrrrrr|}
\hline Concepto de Salud Intercultural & \multicolumn{3}{c}{ Usuario } & \multicolumn{4}{c|}{ Proveedor } & P \\
& Moda & $\bar{x}$ & DS & Moda & $\bar{x}$ & DS & \\
\hline Integrar prácticas de medicina mapuche & 5 & 4,2 & 0,9 & & 2 & 4,0 & 0,9 & 0,11 \\
Incorporar agentes tradicionales & 5 & 3,5 & 1,3 & 4 & 3,2 & 1,2 & 0,08 \\
Incorporar lengua mapuche & 5 & 4,2 & 0,9 & & 4 & 3,2 & 1,2 & 0,00 \\
Atención preferencial & 5 & 3,8 & 1,2 & & 2 & 2,5 & 1,2 & 0,00 \\
Uso de facilitador intercultural & 5 & 4,6 & 0,6 & & 5 & 4,2 & 1,0 & 0,00 \\
\hline
\end{tabular}


$58 \%$ de la varianza ( $\alpha=0.69)$ indica que incorporar la lengua mapuche y a los facilitadores interculturales en el sistema de atención de la población mapuche es una expresión de interculturalidad.

3. Elementos simbólicos y prácticos necesarios para el desarrollo de una política de salud intercultural en la IX región. Usuarios mapuches y proveedores concuerdan que los elementos simbólicos más importantes para desarrollar una política de salud intercultural (Tabla 3), son aquellos relacionados con aumentar el conocimiento sobre la cultura mapuche, promover el respeto a la diversidad cultural y tener una actitud de flexibilidad y aceptación de las diferencias. Como aspectos prácticos los participantes sugieren que la política de salud intercultural debería establecer sistemas de derivación y coordinación con agentes de la medicina tradicional mapuche, y procurar un espacio dentro del sistema de salud para la atención de agentes tradicionales. Estos resultados concuerdan con el análisis de componentes principales en que sólo dos factores explican el $55 \%(\alpha=0,73)$ y $51 \%$ $(\alpha=0,67)$ de la varianza respectivamente. El primer factor identifica como elementos ejes para el desarrollo de una política de salud intercultural, el respeto y actitud flexible frente a las prácticas de salud de la cultura mapuche, y el segundo factor señala que se deben establecer sistemas de derivación hacia agentes de la medicina tradicional, y permitir la atención de éstos al interior del sistema biomédico de salud.

4. Principios de gestión para la implementación de la política de salud intercultural en la IX región. La «gestión en política de salud intercultural», se refiere a los elementos necesarios para desarrollar una política en salud intercultural y considera la participación, la autogestión, la pertinencia cultural y la difusión. En proveedores como en usuarios predo-
Tabla 3. Elementos constituyentes de una política de salud intercultural. D istribución de la opinión de acuerdo a proveedores y usuarios en la IX región

\begin{tabular}{|lccc|}
\hline Categoría & $\begin{array}{c}\text { Proveedor } \\
\%\end{array}$ & $\begin{array}{c}\text { Usuario } \\
\%\end{array}$ & $\mathrm{p}$ \\
\hline Conocimiento & 93,75 & 93,73 & 0,498 \\
Respeto & 79,69 & 90,23 & 0,975 \\
Actitud & 93,75 & 95,24 & 0,676 \\
Derivación & 39,06 & 64,91 & 0,999 \\
Agente tradicional & 34,38 & 74,44 & 1,000 \\
Espacio mapuche & 35,94 & 50,63 & 0,986 \\
Ceremonias & 39,06 & 54,64 & 0,989 \\
\hline
\end{tabular}

minan: la difusión de la política, referida al reconocimiento en todos los centros de salud; el proceso de autogestión y participación de las organizaciones indígenas, relacionada con el diseño y ejecución de los programas de salud que deben incorporar representantes de las comunidades y organizaciones mapuches; y la pertinencia cultural, entendida como la incorporación de creencias y costumbres de la cultura mapuche (Tabla 4).

Este dominio extrajo un solo componente que explica el 57\% de la varianza $(\alpha$ de Cronbach $=$ 0,72 ). La gestión en salud intercultural en la región estaría asociada a la necesidad de otorgar una mayor participación a las organizaciones indígenas en la elaboración de planes y programas de salud pertinentes a la cultura mapuche, vinculado a procesos de autogestión de las comunidades, y una permanente difusión de la política de salud intercultural en todos los niveles de atención.

5. Desafíos regionales para una política de salud intercultural. En los desafíos regionales para el desarrollo e implementación de una política de salud intercultural, se observa en la

Tabla 4. Elementos de gestión en política de salud intercultural. D istribución de la opinión de acuerdo a proveedores y usuarios en la IX región

\begin{tabular}{|lccccccccc|}
\hline Elementos de Gestión & \multicolumn{3}{c}{ Usuario } & \multicolumn{4}{c}{ Proveedor } & P \\
& Moda & $\bar{x}$ & DS & & Moda & $\bar{x}$ & DS & \\
\hline Participación de las organizaciones indígenas & 5 & 4,2 & 0,8 & & 4 & 3,3 & 1,2 & 0,00 \\
Proceso de autogestión & 4 & 3,6 & 1,2 & & 2 & 2,8 & 1,3 & 0,00 \\
Pertinencia cultural & 5 & 4,2 & 0,9 & & 4 & 4,1 & 0,9 & 0,21 \\
Difusión de la política & 5 & 4,4 & 0,7 & & 4 & 3,8 & 1,1 & 0,00 \\
\hline
\end{tabular}


Tabla 5, que el primer elemento relevante es discusión entre autoridades de gobierno y organizaciones mapuches para acordar un programa; el segundo, es la falta de capacitación de los equipos y desconocimiento de la cultura mapuche, referido a que el equipo de salud no está capacitado, bajó un programa educativo formal, en el tema de la cultura mapuche. Se observan diferencias significativas entre usuarios y proveedores, en que los usuarios mapuches consideran un desafío importante enfrentar la falta de voluntad, la indiferencia y discriminación de los equipos de salud con el tema de la cultura mapuche, sin embargo, los proveedores plantean no estár de acuerdo ( $\mathrm{p}: 0,00$ ).

En el análisis de los componentes principales, este dominio es explicado por dos factores. El primer factor $(\alpha=0,76)$ que explica el $78 \%$ de la varianza identifica la falta de voluntad de las autoridades y la indiferencia de los equipos de salud como un desafío importante en la gestión de una política de salud intercultural en la región. El segundo factor $(a=0,67)$ y $51 \%$ del total de la varianza indica que la falta de capacitación de los equipos, desconocimiento sobre la cultura mapuche, y la escasa formación de los estudiantes en la Facultad de Medicina en la temática de salud y cultura mapuche corresponden a un segundo grupo de problemas que enfrenta el desarrollo de una política de salud intercultural.

\section{DisCUSIÓN}

Los resultados de este estudio, demuestran importantes desacuerdos entre proveedores de salud y usuarios mapuches, respecto a temas centrales como son la necesidad y justificación de una política de salud intercultural. Para los usuarios la falta de acceso, su condición étnica y la inequidad en la atención de salud son razones fundamentales para crear una política que supere estos problemas desde una perspectiva cultural. Los proveedores en cambio, no consideran que la población mapuche tenga desventajas comparativas frente al resto de la población usuaria del sistema de salud, por lo tanto, no justifican el desarrollo de una política de salud para la población indígena.

Una segunda área de desacuendo dice relación con los desafíos regionales para desarrollar la política de salud intercultural. Mientras los usuarios mapuches opinaron que la falta de voluntad de los directivos de salud, la indiferencia de la población frente a la problemática mapuche, y la discriminación de los equipos de salud hacia la población indígena constituyen los principales problemas en la región; los proveedores opinaron que éstos son la falta de capacitación y conocimiento sobre cultura mapuche, y la escasa formación en el tema que se proporciona en las universidades.

Diversos estudios con pueblos indígenas acerca de la relación entre cultura y medicina, demuestran que las múltiples divergencias entre usuarios y proveedores emergen en primer lugar, por la presencia de diferentes modelos explicativos de salud y enfermedad de los grupos en contacto, y en segundo lugar por las condiciones de dominación social y política de un modelo sobre otro ${ }^{4-6}$.

Un elemento de reflexión lo plantea Walsh ${ }^{7}$, respecto a la interculturalidad, referida como un proceso permanente de relación, comunicación, conocimiento, valores y tradiciones distintas orien-

\section{Tabla 5. D esafíos regionales para una política de salud intercultural. D istribución de la opinión de acuerdo a proveedores y usuarios en la IX región}

\begin{tabular}{|lcccccccc|}
\hline \multirow{2}{*}{ Desafíos regionales } & \multicolumn{3}{c}{ Usuario } & \multicolumn{5}{c|}{ Proveedor } \\
& Moda & $\overline{\mathrm{x}}$ & DS & & Moda & $\overline{\mathrm{x}}$ & DS \\
\hline \multirow{2}{*}{ Falta de voluntad } & 4 & 3,7 & 1,1 & & 2 & 2,6 & 1,1 & 0,000 \\
Indiferencia de equipos de salud & 4 & 3,4 & 1,2 & & 2 & 2,6 & 1,0 & 0,000 \\
Discusión & 4 & 4,1 & 0,8 & 4 & 3,6 & 1,0 & 0,000 \\
Discriminación & 4 & 3,9 & 0,9 & 2 & 2,8 & 1,2 & 0,000 \\
Falta capacitación de los equipos & 4 & 4,1 & 0,8 & 4 & 3,9 & 0,9 & 0,163 \\
Escasa formación en universidad & 3 & 3,4 & 1,0 & 4 & 3,6 & 0,9 & 0,920 \\
Desconocimiento de la cultura mapuche & 4 & 3,8 & 1,0 & & 4 & 3,5 & 1,1 & 0,013 \\
\hline
\end{tabular}


tadas a generar, construir y propiciar un respeto mutuo y un desarrollo pleno de las capacidades de los individuos por encima de sus diferencias culturales y sociales. Este razonamiento tiene relación con el concepto de salud intercultural de usuarios y proveedores, ya que para los proveedores está centrada en aspectos simbólicos de la cultura, tales como: respeto a la identidad, aumentar el conocimiento. En cambio, para los usuarios, además de estos aspectos considera necesario la manifestación concreta en acciones tales como: incorporar a la machi, derivación de los enfermos, y un espacio mapuche para la atención.

Es importante tener en cuenta las áreas de consenso de usuarios y proveedores, referidas a la realidad de poseer una política de salud intercultural en que las acciones y programas deben ser pertinentes a la cultura mapuche y una vez que se establezcan debe generarse con la participación de organizaciones indígenas y ampliamente difundidas en el sistema de salud.

Al respecto Avaria ${ }^{8}$, señala que los pueblos indígenas demandan respeto y ejercicio real de procesos participativos orientados al reconocimiento de éstos, como elaboradores, articuladores y gestionadores de sus procesos de desarrollo y de participación política.

Por otra parte, queda planteado el concepto básico de interculturalidad en la IX región, dado

\section{REFERENCIAS}

1. Citarella L, Conejeros A, Espinoza B, Jelves I, Oyarce AM, Vidal A et al. Medicinas y cultura en la Araucanía. 2a edición. Santiago. Chile. Editorial Sudamericana. 2000.

2. Alarcón A, Vidal A, Neira J. Salud Intercultural: Elementos para la construcción de sus bases conceptuales. Rev Méd Chile 2003; 131: 1061-5.

3. O.P.S. Salud de los pueblos indígenas de las Améncas. Rev Panam Salud Publica 1998; 3: 278-84.

4. Ministerio de Salud. Política de salud y pueblos indígenas. Documento del Gobierno de Chile. Ministerio de salud. División de Rectoría y Regulación Sanitaria. Enero. 2003.

5. Kieinman A. Patients and healers in the context of culture. An exploration of the borderland between anthropology, medicine and psychiatry. por la expresión de la salud a través de la lengua e integración de prácticas de la medicina, además se agregan otros elementos básicos que lo constituyen como el conocimiento de la cultura y respeto a las prácticas y costumbres mapuches. Al respecto, Citarella et $\mathrm{al}^{1}$, señala que ésta apunta al fortalecimiento y libre desarrollo de los sistemas médicos culturales en el marco del mantenimiento de la pluralidad étnica nacional y de una superación de la condición de marginalidad en la cual se encuentran los pueblos indígenas.

Finalmente, respecto de la interacción entre los sistemas de atención médica y la comunicación intercultural, autores como Green ${ }^{6}$; Herman ${ }^{9}$, señalan que los procesos de interacción social y cultural que involucren la existencia de diferentes y, a veces, antagónicos sistemas de creencias están sujetos a importantes fricciones. En esta misma línea Berlin y Fowkes ${ }^{10}$, señalan: el éxito de la comunicación intercultural puede ser logrado a través de un proceso que incluya cinco elementos: escuchar, explicar, reconocer, recomendar y negociar.

El presente estudio proporcionará información válida y confiable como referente teónico respecto al significado de la interculturalidad en salud a partir de la población mapuche y actores de salud occidental. Ello permitirá avanzar hacia el desarrollo y promoción de un sistema de salud intercultural basado en las expectativas de la población en la región.
University of California Press USA. 1984.

6. Green EC. The integration of Modem and Tradicional Health Sectors in Swaziland. En: Antropological Praxis Robert M, Wulff SJ, Fiske, eds. Wesview Press Boulder. London. 1997; 87-97. 1987.

7. Walsh C. Propuesta para el tratamiento de la interculturalidad en la educación. UNEBI-MED. Lima. Perú. 2000.

8. Avaria A. Políticas sociales: exclusión/inclusión del mundo indígena. Rev Mad 2003; 8: 1-17. Disponible en: http://rehue.csociales.uchile.cl/ publicaciones/mad/08/paper05.htm. [Consultado el 13 de enero del 2004].

9. Helman CG. Culture, Health and Iones. Butterworth-Heinemann. Oxford. 1994.

10. BERLiN E, FowKes C. Teaching Framework for Cross-Cultural Health Care. Mayfield Publishing Company. California. 1998. 